\title{
ON CERTAIN WALLMAN SPACES
}

\author{
ROY CONNELL \\ Department of Academic Skills and Mathematics \\ Hunter College \\ The City University of New York \\ 695 Park Avenue \\ New York, NY 10021 \\ (Received October 15, 1992 and in revised form April 7, 1993)
}

ABSTRACT. Several generalized Wallman type spaces are considered as well as various lattices of subsets therein. In particular, regularity of these lattices and consequences are investigated. Also considered are necessary and sufficient conditions for these lattices to be Lindelöf as well as replete. prime complete, and fully replete.

KEY WORDS AND PHRASES. Zero-one valued measures, $\sigma$-smooth measures, lattice properties, regularity, normal, disjunctive, and replete.

1980 AMS SUBJECT CLASSIFICATION CODE. 28C15, 28 A60.

\section{INTRODUCTION.}

Let $X$ be an arbitrary set and $\mathcal{L}$ a lattice of subsets of $X$ such that $\emptyset, X \in \mathcal{L}$. $\mathcal{A}(\mathcal{L})$ designates the algebra generated by $\mathcal{L}$, and $I(\mathcal{L})$ is the set of zero-one valued finitely additive measures on $\mathcal{A}(\mathcal{L})$.

$I_{\sigma}(\mathcal{L})$ denotes those elements of $I(\mathcal{L})$ that are $\sigma$-smooth on $\mathcal{L}$, and $I^{\sigma}(\mathcal{L})$ those which are $\sigma$-smooth on $\mathcal{A}(\mathcal{L})$ i.e. are countably additive. $I_{R}(\mathcal{L})$ denotes those elements of $I(\mathcal{L})$ which are $\mathcal{L}$-regular; while $I_{R}^{\sigma}(\mathcal{L})$ denotes those elements of $I_{R}(\mathcal{L})$ which are also in $I_{\sigma}(\mathcal{L})$, and consequently in $I^{\sigma}(\mathcal{L})$. These various sets of measures have distinguishing lattices of subsets within them which are taken as bases for closed set topologies, and are called generalized Wallman spaces.

Some of these spaces such as $I_{R}(\mathcal{L})$ and $I_{R}^{\sigma}(\mathcal{L})$ where $\mathcal{L}$ is disjunctive are well known and in special cases give known topological spaces.

We wish to investigate in some detail the spaces $I_{\sigma}(\mathcal{L})$ and $I^{\sigma}(\mathcal{L})$ and the lattices of these spares (see below for explicit definitions). In particular, we investigate when these lattices are regular and subsequent consequences of regularity. We also find necessary and sufficient conditions for these spaces to be Lindelö and also, for a number of these lattices, consider questions of repleteners prime completeness and fully repleteness.

We begin in section 2 with some background information on these spaces and lattices and summarize a variety of known results. Then in section 3 and 4 we pursue our investigation an indicated above.

The author takes pleasure in acknowledging his indebtedness to the referee for greatly improving the presentation of this paper.

\section{BACKGROUND AND NOTATIONS.}

We follow standard notation and terminology such as appears in $[1,2,4,6,11]$. We review some of this here for the reader's convenience.

Let $X$ be a set and $\mathcal{L}$ a lattice of subsets of $X$ such that $\emptyset, X$ belong to $\mathcal{L} . \mathcal{A}(\mathcal{L})$ denotes the algebra generated by $\mathcal{L}$, and $M(\mathcal{L})$ denotes those finitely additive, bounded, non-negative measures on $\mathcal{A}(\mathcal{L})$ which are non-trivial. $M_{\sigma}(\mathcal{L})$ denotes these elements of $M(\mathcal{L})$ which are $\sigma$-smooth on $\mathcal{L}$. That is, $\mu \in M_{\sigma}(\mathcal{L})$, if for $L_{n} \in \mathcal{L}$ and $L_{n} \downarrow \emptyset$, we have $\mu\left(L_{n}\right) \rightarrow 0 . M^{\sigma}(\mathcal{L})$ denotes these elements of $M(\mathcal{L})$ which are $\sigma$-smooth on $\mathcal{A}(\mathcal{L})$ or equivalently here, are countably additive. 
Next, $M_{R}(\mathcal{L})$ denotes these $\mu \in M(\mathcal{L})$ which are $\mathcal{L}$-regular. That is $\mu(A)=\sup \{\mu(L) \mid$ $L \subset A, L \in \mathcal{L}$ \} where $A \in \mathcal{A}(\mathcal{L})$. Note, if $\mu \in M_{R}(\mathcal{L})$ and $\mu \in M_{\sigma}(\mathcal{L})$ then $\mu \in M^{\sigma}(\mathcal{L})$ Such measures are denoted by $M_{R}^{\sigma}(\mathcal{L})$. Finally the zero-one valued subsets of the above are denoted by $I(\mathcal{L}) . I_{\sigma}(\mathcal{L}), I^{\sigma}(\mathcal{L}), I_{R}(\mathcal{L})$ and $I_{R}^{\sigma}(\mathcal{L})$ respectively.

If $\mu \in M(\mathcal{L})$, the support of $\mu, S(\mu)$ is defined as follows. $S(\mu)=\cap\{L \in \mathcal{L} \mid \mu(L)=\mu(X)\}$. We recall (see [2,6,9]). $\mathcal{L}$ is compact if and only if $S(\mu) \neq \emptyset$ for all $\mu \in I(\mathcal{L})$, or equivalently $S(\mu) \neq \emptyset$ for all $\mu \in I_{R}(\mathcal{L})$, or equivalently $S(\mu) \neq \emptyset$ for all $\mu \in \Pi(\mathcal{L})$. Here, $\pi \in \Pi(\mathcal{L})$ if $\pi: \mathcal{L} \rightarrow\{0,1\}, \pi(X)=1$, and $\pi$ is monotone, and $\pi(A \cap B)=\pi(A) \pi(B), A, B \in \mathcal{L}$, and $\pi(\theta)=0 . \Pi_{\sigma}(\mathcal{L})$ denotes these $\pi \in \Pi(\mathcal{L})$ which are $\sigma$-smooth on $\mathcal{L}$. For $\pi \in \Pi(\mathcal{L}), S(\pi)$ is defined as for $\mu \in I(\mathcal{L})$. With these definitions in place we have: $\mathcal{L}$ is countably compact (c.c.) if and only if $I(\mathcal{L})=I_{\sigma}(\mathcal{L})$, equivalently $I_{R}(\mathcal{L})=I_{R}^{\sigma}(\mathcal{L})$, or $\Pi(\mathcal{L})=\Pi_{\sigma}(\mathcal{L}) . \mathcal{L}$ is Lindelöf if and only if for all $\pi \in \Pi_{\sigma}(\mathcal{L}), S(\pi) \neq \emptyset$. $\mathcal{L}$ is replete if $S(\mu) \neq \emptyset$ for all $\mu \in I_{R}^{\sigma}(\mathcal{L})$. $\mathcal{L}$ is prime complete if $S(\mu) \neq \emptyset$ for all $\mu \in I_{\sigma}(\mathcal{L})$, and $\mathcal{L}$ is fully replete if $S(\mu) \neq \emptyset$ for all $\mu \in I^{\sigma}(\mathcal{L})$.

We can characterize other more familiar lattice-topological concepts in terms of these measures For example (see [2,6,9]) $\mathcal{L}$ is disjunctive if and only if $\mu_{x} \in I_{R}(\mathcal{L})$ for all $x \in X$, where $\mu_{x}$ is the Dirac measure concentrated at $x . \mathcal{L}$ is regular if and only if for $\mu_{1}, \mu_{2} \in I(\mathcal{L})$ with $\mu_{1} \leq \mu_{2}(\mathcal{L})$, that is $\mu_{1}(L) \leq \mu_{2}(L)$ for all $L \in \mathcal{L}$ we have $S\left(\mu_{1}\right)=S\left(\mu_{2}\right)$.

$\mathcal{L}$ is $T_{2}$ if and only if $S(\mu)=\emptyset$ or a singleton for all $\mu \in I(\mathcal{L})$, and $\mathcal{L}$ is normal if and only if for $\mu \leq \nu_{1}(\mathcal{L}), \mu \leq \nu_{2}(\mathcal{L})$ where $\mu \in I(\mathcal{L}), \nu_{1}, \nu_{2} \in I_{R}(\mathcal{L})$, we have $\nu_{1}=\nu_{2}$. Finally, we note that various sets of zero-one valued measures on $\mathcal{A}(\mathcal{L})$ can be topologized using Wallman topologies. Let $I$ be any subset of $I(\mathcal{L})$, e.g. $I_{R}(\mathcal{L}), I_{\sigma}(\mathcal{L})$, etc. Let $A \in \mathcal{A}(\mathcal{L})$, and denote by $H(A)=\{\mu \in I \mid \mu(A)=1\}$. Then for $A, B \in \mathcal{A}(\mathcal{L})$ we have: $H(A \cup B)=H(A) \cup H(B), H(A \cap B)=$ $H(A) \cap H(B), A \supset B$ implies $H(A) \supset H(B)$. If $\left\{\mu_{x}: x \in X\right\} \subset I$. Then $H(A) \supset H(B)$ implies $A \supset B$. Also, $I-H(A)=H\left(A^{\prime}\right)$ where throughout the prime will designate the complementary set. The Wallman topology on $I$ is obtained by taking the set of all $H(L), L \in \mathcal{L}$ as a base for the closed sets. In the particular cases of $I=I(\mathcal{L}), I_{R}(\mathcal{L}), I_{\sigma}(\mathcal{L}), I^{\sigma}(\mathcal{L}), I_{R}^{\sigma}(\mathcal{L})$ denote these bases by $V(\mathcal{L}), W(\mathcal{L}) V_{\sigma}(\mathcal{L}), V^{\sigma}(\mathcal{L}), W_{\sigma}(\mathcal{L})$ respectively. Some of these spaces have been thoroughly investigated. In particular $I_{R}(\mathcal{L}), \tau W(\mathcal{L})(\tau \mathcal{L}$ in general denotes the lattice of arbitrary intersection. of sets of $\mathcal{L}$ ) which is a compact $T_{1}$ space, and assuming $\mathcal{L}$ is disjunctive then it is $T_{2}$ if and only if $\mathcal{L}$ is normal. $I(\mathcal{L}), \tau V(\mathcal{L})$ is compact $T_{0}$. If $\mathcal{L}$ is disjunctive then the lattice $W_{\sigma}(\mathcal{L})$ in $I_{R}^{o}(\mathcal{L})$ i replete. We propose to investigate further topological properties of these general Wallman ipaim and to relate some of these topological properties to the underlying lattice $\mathcal{L}$.

Related material can be found in $[4,7,10]$. A few of these results will be cited in the next wertun to show how it interrelates with our work.

\section{TOPOLOGICAL PROPERTIES OF THE WALLMAN SPACES.}

We note first some known facts about various Wallman spaces, (see $[2,9,11,12]) . W(\mathcal{L})$ is a compact lattice in $I_{R}(\mathcal{L}), V(\mathcal{L})$ is a compact lattice in $I(\mathcal{L})$. If $\mathcal{L}$ is disjunctive, then $W_{\sigma}(\mathcal{L})$ is replete in $I_{R}^{\sigma}(\mathcal{L}) . V_{\sigma}(\mathcal{L})$ is prime complete in $I_{\sigma}(\mathcal{L})$, and $V^{\sigma}(\mathcal{L})$ is fully replete in $I^{\sigma}(\mathcal{L})$. Moreover, we have (see [8]).

THEOREM 3.1. $I(\mathcal{L}), V(\mathcal{L})$ is compact $T_{0}$. Also, $V(\mathcal{L})$ is $T_{1}$ if and only if $I(\mathcal{L})=I_{R}(\mathcal{L})$, which is equivalent to $V(\mathcal{L})$ being disjunctive and is also equivalent to $V(\mathcal{L})$ being regular.

REMARK. $I(\mathcal{L})=I_{R}(\mathcal{L})$ is equivalent to $\mathcal{L}$ being an algebra. Various proof of this appear in $[8,9]$. It is even true for abstract distributive lattice (see [3]).

For the pair $I_{R}(\mathcal{L}), W(\mathcal{L})$ we have $([7])$.

THEOREM 3.2. If $\mathcal{L}$ is disjunctive then the following are equivalent:

(1) $W(\mathcal{L})$ is normal.

(2) $W(\mathcal{L})$ is regular.

(3) $W(\mathcal{L})$ is $T_{2}$. 
REMARK: Since $W(\mathcal{L})$ is compact, $\tau W(\mathcal{L})$ is compact and $I_{R}(\mathcal{L}), \tau W(\mathcal{L})$ gives well-known compactifications of $X$ in the case where $X$ is a topological space, and $\mathcal{L}$ consist of closed sets, zero sets, clopen sets, etc.

We consider now $W_{\sigma}(\mathcal{L})$ in $I_{R}^{\sigma}(\mathcal{L})$. If $\mathcal{L}$ is disjunctive, then $W_{\sigma}(\mathcal{L})$ is replete. It is prime complete if and only if

(3.1) For any $\mu \in I_{\sigma}(\mathcal{L})$ there exist a $\nu \in I_{R}^{\sigma}(\mathcal{L})$ such that $\mu \leq \nu(\mathcal{L})$. See [10]. Also if we consider $I_{R}^{\sigma}(\mathcal{L})$ with the topology of closed sets $\tau W_{\sigma}(\mathcal{L})$ then, if $\mathcal{L}$ is disjunctive, $I_{R}^{\sigma}(\mathcal{L}), \tau W_{\sigma}(\mathcal{L})$ is Lindelöf if and only if

(3.2) For any $\pi \in \Pi_{\sigma}(\mathcal{L})$ there exist a $\mu \in I_{R}^{\sigma}(\mathcal{L})$ such that $\pi \leq \mu(\mathcal{L})$.

In a similar manner, considering the pair $I_{\sigma}(\mathcal{L})$ and $V_{\sigma}(\mathcal{L})$, we have that the topological space $I_{\sigma}(\mathcal{L}), \tau V_{\sigma}(\mathcal{L})$ is Lindelö if and only if

(3.3) For any $\pi \in \Pi_{\sigma}(\mathcal{L})$ there exist a $\mu \in I_{\sigma}(\mathcal{L})$ such that $\pi \leq \mu(\mathcal{L})$. Again see [10] for details.

We further note (details can be found in [5]).

THEOREM 3.3. If $\mathcal{L}$ is disjunctive, then in $I_{R}^{\sigma}(\mathcal{L})$ the lattice $W_{\sigma}(\mathcal{L})$ is fully replete if and only if

(3.4) For any $\mu \in I^{\sigma}(\mathcal{L})$ there exists a $\nu \in I_{R}^{\sigma}(\mathcal{L})$ such that $\mu \leq \nu(\mathcal{L})$.

We now consider the pair $I^{\sigma}(\mathcal{L})$ and $V^{\sigma}(\mathcal{L})$.

THEOREM 3.4. Consider the condition

(3.5) For any $\pi \in \Pi_{\sigma}(\mathcal{L})$ there exist a $\mu \in I^{\sigma}(\mathcal{L})$ such that $\pi \leq \mu(\mathcal{L})$.

Then we have

1) If (3.5) holds and if $\mathcal{L}$ is fully replete, then $\mathcal{L}$ is Lindelöf.

2) If $\mathcal{L}$ is Lindelöf then $\mathcal{L}$ satisfies (3.5).

3) $\mathcal{L}$ satisfies (3.5) if and only if the topological spaces $I^{\sigma}(\mathcal{L}), \tau V^{\sigma}(\mathcal{L})$ is Lindelöf.

4. FURTHER PROPERTIES OF THE LATTICES $W_{\sigma}(\mathcal{L}), V_{\sigma}(\mathcal{L})$ and $V^{\sigma}(\mathcal{L})$.

It has been shown in [7] that if $\mathcal{L}$ is disjunctive, then $W_{\sigma}(\mathcal{L})$ is regular if and only if the following condition holds.

(4.1) For all $\mu_{1}, \mu_{2} \in I(\mathcal{L})$ and $\nu \in I_{R}^{\sigma}(\mathcal{L}) \mu_{1} \leq \mu_{2}(\mathcal{L})$ and $\mu_{1} \leq \nu(\mathcal{L})$, then $\mu_{2} \leq \nu(\mathcal{L})$ It w conditions of this type that we wish to consider next.

We first note that in dealing with the various Wallman spaces, I, (see section 2) a bijection can be set up between elements of I and zero-one valued measures on $H\left(A(\mathcal{L})\right.$ ) by defining $\mu^{\prime}$ on $H(A(\mathcal{L}))$ by $\mu^{\prime}(H(A))=\mu(A)$, where $A \in \mathcal{A}(\mathcal{L})$, and $\mu \in I$. In order to establish this bijection in the case $I=I_{R}(\mathcal{L})$ or $I_{R}^{\sigma}(\mathcal{L})$, and $H(\mathcal{L})=W(\mathcal{L})$ or $W_{\sigma}(\mathcal{L})$ respectively, we must assume that $\mathcal{L}$ is disjunctive. In what follows $I \neq I_{R}(\mathcal{L})$ or $I_{R}^{\sigma}(\mathcal{L})$, and it will not be necessary to assume that $\mathcal{L}$ is disjunctive.

THEOREM 4.1. Consider $I_{\sigma}(\mathcal{L}), V_{\sigma}(\mathcal{L})$. Then $V_{\sigma}(\mathcal{L})$ is regular if and only if the following holds:

(4.2) For all $\mu_{1}, \mu_{2} \in I(\mathcal{L})$ and $\nu \in I_{\sigma}(\mathcal{L})$ if $\mu_{1} \leq \mu_{2}(\mathcal{L})$ and $\mu_{1} \leq \nu(\mathcal{L})$, then $\mu_{2} \leq \nu(\mathcal{L})$.

PROOF. Suppose condition (4.2) holds, then we wish to show that $V_{\sigma}(\mathcal{L})$ is regular. Let $\mu_{1}^{\prime}, \mu_{\iota}^{\prime} \in I\left(V_{\sigma}(\mathcal{L})\right)$, and $\mu_{1}^{\prime} \leq \mu_{2}^{\prime}\left(V_{\sigma}(\mathcal{L})\right)$ where $\mu_{1}, \mu_{2} \in I(\mathcal{L})$. Then $S\left(\mu_{2}^{\prime}\right) \subset S\left(\mu_{1}^{\prime}\right)$.

If $\lambda \in S\left(\mu_{1}^{\prime}\right)$ then $\lambda \in I_{\sigma}(\mathcal{L})$ and $\mu_{1} \leq \lambda(\mathcal{L})$. But $\mu_{1}^{\prime} \leq \mu_{2}^{\prime}\left(V_{\sigma}(\mathcal{L})\right)$ implies $\mu_{1} \leq \mu_{2}(\mathcal{L})$. Thus by condition (4.2) $\mu_{2} \leq \lambda(\mathcal{L})$. Hence $\lambda \in S\left(\mu_{2}^{\prime}\right)$. Thus $S\left(\mu_{2}^{\prime}\right)=S\left(\mu_{1}^{\prime}\right)$, and $V_{\sigma}(\mathcal{L})$ is regular.

Conversely, suppose $V_{\sigma}(\mathcal{L})$ is regular. Let $\mu_{1}, \mu_{2} \in I(\mathcal{L})$ and $\lambda \in I_{\sigma}(\mathcal{L}), \mu_{1} \leq \mu_{2}(\mathcal{L})$ and $\mu_{1} \leq \nu(\mathcal{L})$. Then $\mu_{1}^{\prime} \leq \mu_{2}^{\prime}\left(V_{\sigma}(\mathcal{L})\right)$, and $\mu_{1}^{\prime} \leq \nu^{\prime}\left(V_{\sigma}(\mathcal{L})\right)$. Since $V_{\sigma}(\mathcal{L})$ is regular, we have $S\left(\mu_{1}^{\prime}\right)=$ $S\left(\mu_{2}^{\prime}\right)=S\left(\nu^{\prime}\right)$. But $\nu \in S\left(\nu^{\prime}\right)$. So $\nu \in S\left(\mu_{2}^{\prime}\right)$, and, therefore, $\mu_{2} \leq \nu(\mathcal{L})$. Thus condition (4.2) holds.

Using this result we can prove the following:

THEOREM 4.2. $I_{\sigma}(\mathcal{L}), V_{\sigma}(\mathcal{L})$ is regular if and only $I_{\sigma}(\mathcal{L})=I_{R}^{\sigma}(\mathcal{L})$.

PROOF. Let $\mu \in I_{\sigma}(\mathcal{L})$. There exists a $\nu \in I_{R}(\mathcal{L})$ such that $\mu \leq \nu(\mathcal{L})$. Therefore $\mu^{\prime} \leq$ $\nu^{\prime}\left(V_{\sigma}(\mathcal{L})\right)$ where $\mu^{\prime}, \nu^{\prime} \in I\left(V_{\sigma}(\mathcal{L})\right)$. But $S\left(\mu^{\prime}\right)=S\left(\nu^{\prime}\right)$ since $V_{\sigma}(\mathcal{L})$ is regular. Now $\mu \in S\left(\mu^{\prime}\right)$. So 
$\mu \in S\left(\nu^{\prime}\right)$. Thus $\nu \leq \mu(\mathcal{L})$. Hence $\mu=\nu$ and consequently $\mu \in I_{R}^{\sigma}(\mathcal{L})$, and $I_{\sigma}(\mathcal{L})=I_{R}^{\sigma}(\mathcal{L})$.

Conversely, suppose $I_{\sigma}(\mathcal{L})=I_{R}^{\sigma}(\mathcal{L})$. Then $V_{\sigma}(\mathcal{L})=W_{\sigma}(\mathcal{L})$. Now let $\mu_{1}, \mu_{2} \in I(\mathcal{L}), \nu \in I_{\sigma}(\mathcal{L})$ and $\mu_{1} \leq \mu_{2}(\mathcal{L}), \mu_{1} \leq \nu(\mathcal{L})$.

Then since $I_{\sigma}(\mathcal{L})=I_{R}^{\sigma}(\mathcal{L}), \mu_{1} \in I_{R}^{\sigma}(\mathcal{L})$. So $\mu_{1}=\mu_{2}$ and $\mu_{1}=\nu$. Hence condition (4.2) holds and $I_{\sigma}(\mathcal{L})$ is regular.

For the pair $I^{\sigma}(\mathcal{L}), V^{\sigma}(\mathcal{L})$, we have;

THEOREM 4.3. In $I^{\sigma}(\mathcal{L})$, if $V^{o}(\mathcal{L})$ ss regular, then $I^{\sigma}(\mathcal{L})=I_{R}^{\sigma}(\mathcal{L})$.

PROOF. Let $\mu \in I^{\sigma}(\mathcal{L})$ and $\mu \leq \nu(\mathcal{L})$ where $\nu \in I_{R}(\mathcal{L})$. Then $\mu^{\prime} \leq \nu^{\prime}\left(V^{\sigma}(\mathcal{L})\right)$ where $\mu^{\prime}, \iota^{\prime} \in I\left(V^{\sigma}(\mathcal{L})\right)$. Thus $S\left(\mu^{\prime}\right)=S\left(\nu^{\prime}\right)$ since $V^{\sigma}(\mathcal{L})$ is regular. But $\mu \in S\left(\mu^{\prime}\right)$. So $\mu \in S\left(\nu^{\prime}\right)$ and $\nu^{\prime} \leq \mu(\mathcal{L})$. Hence $\mu=\nu \in I_{R}(\mathcal{L})$ and therefore $\mu \in I_{R}^{\sigma}(\mathcal{L})$. Consequently $I^{\sigma}(\mathcal{L})=I_{R}^{\sigma}(\mathcal{L})$.

REMARK. If $I^{\sigma}(\mathcal{L})=I_{R}^{\sigma}(\mathcal{L})$ then it does not necessarily follow as in the case of $I_{\sigma}(\mathcal{L})$ and $V_{\sigma}(\mathcal{L})$ that $V^{\sigma}(\mathcal{L})$ is regular. To prove this we would have to consider the following condition aralogous to $(4.2)$, namely

(4.3) For all $\mu_{1}, \mu_{2} \in I(\mathcal{L})$ and $\nu \in I^{\sigma}(\mathcal{L})$ if $\mu_{1} \leq \mu_{2}(\mathcal{L})$ and $\mu_{1} \leq \nu(\mathcal{L})$. Then $\mu_{2} \leq \nu(\mathcal{L})$.

The problem in verifying this condition is that if $\mu_{1} \leq \nu(\mathcal{L})$, then it only follows that $\mu_{1} \in I_{\sigma}(\mathcal{L})$, and one cannot say that $\mu_{1} \in I_{R}^{\sigma}(\mathcal{L})$ as we could in the case of $I_{\sigma}(\mathcal{L})$ and $V_{\sigma}(\mathcal{L})$.

Finally we consider a refinement of condition (3.1), namely:

(4.4) For any $\mu \in I_{\sigma}(\mathcal{L})$ there exists a $\nu \in I^{\sigma}(\mathcal{L})$ such that $\mu \leq \nu(\mathcal{L})$.

THEOREM 4.4. $\mathcal{L}$ satisfies condition $(4.4)$ if and only if $I^{\sigma}(\mathcal{L}), V^{\sigma}(\mathcal{L})$ is prime complete.

PROOF. Let $\lambda \in I_{\sigma}\left(V^{\sigma}(\mathcal{L})\right)$. Then $\lambda=\mu^{\prime}$ where $\mu \in I_{\sigma}(\mathcal{L})$, and by (4.4) there exists a $\nu^{\prime} \in I^{\sigma}(\mathcal{L})$ such that $\mu \leq \nu(\mathcal{L})$. Hence $\lambda=\mu^{\prime} \leq \nu^{\prime}\left(V^{\sigma}(\mathcal{L})\right)$ and $\nu^{\prime} \in I^{\sigma}\left(V^{\sigma}(\mathcal{L})\right)$. But $V^{\sigma}(\mathcal{L})$ is fully

replete, so $S\left(\nu^{\prime}\right) \neq \emptyset$. Thus $S\left(\mu^{\prime}\right)=S(\lambda) \neq \emptyset$, and $V^{\sigma}(\mathcal{L})$ is prime complete.

Conversely, suppose $V^{\sigma}(\mathcal{L})$ is prime complete. Let $\mu \in I_{\sigma}(\mathcal{L})$. Then $\mu^{\prime} \in I_{\sigma}\left(V^{\sigma}(\mathcal{L})\right)$. Hence, $S\left(\mu^{\prime}\right) \neq \emptyset$ since $V^{\sigma}(\mathcal{L})$ is prime complete. Then there exist a $\nu \in S\left(\mu^{\prime}\right)$ where $\nu \in I^{\sigma}(\mathcal{L})$ and clearly $\mu \leq \nu(\mathcal{L})$. Thus $(4.4)$ is satisfied.

\section{REFERENCES}

[1] Alexandroff, A., Additive set functions in abstract spaces, Mat. $\mathrm{Sb}$ (N.S.), 8,50 (1940), 307348.

[2] Bachman, G. and Stratigos, P. On general lattice, repleteness and completeness, Illinois Jour. of Math. 27, No. 4, (1983), 535-561.

[3] Bourbaki, N., Elements of Mathematics, General Topology, Part 1, Addison Wesley, Reading, Mass. (1966).

[4] Eid, G., On normal lattices and Wallman spaces, Internat., J. Math. and Math. Sci., 13, No. $1,(1990), 31-38$.

[5] Eid, G. On Wallman spaces and the Lindelöf property, General Topology and Applications, Sixth Summer Conference, Annals N.Y. Acad of Sci, 659, NY (1992), 57-62.

[6] Frolik, Z., Prime filters with the C.I.P. Comm. Math. Univ. Carolinae, 13, (1972), 553-575.

[7] Grassi, P., Measure characterizations and properties of normal and regular lattices, Internat. Jour. Math. and Math.-Sci., 14, No. 2, (1991), 385-392.

[8] Schutz, R., On regular and sigma-smooth two valued measures and lattice generated topologies. (To appear).

[9] Szeto, M., On separation of lattices, Internat. Jour. Math. and Math.-Sci., 14, No. 2, (1991), 325-338.

[10] Vlad, C., Lattice separation and properties of Wallman type spaces, Ann. di Mat. pura ed appl., (IV), 155, (1991), 65-79.

[11] Wallman, H., Lattices and topological spaces, Ann. of Math. 39, (1938), 112-126.

[12] Yallaoui, El-B., Repleteness and regular lattice measures, Per. Math. Hungarica, 23(2), (1991), 109-120. 


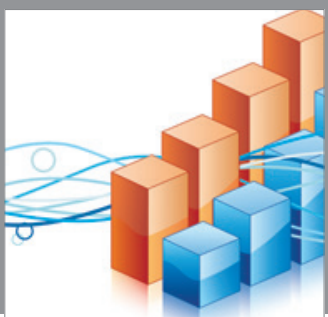

Advances in

Operations Research

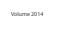

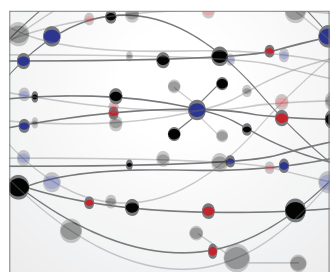

\section{The Scientific} World Journal
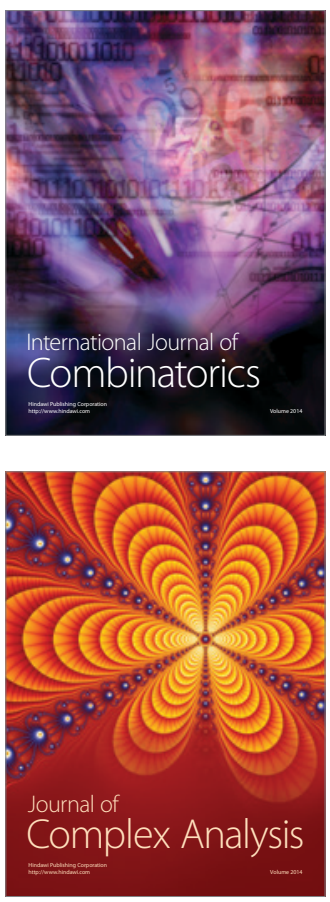

International Journal of

Mathematics and

Mathematical

Sciences
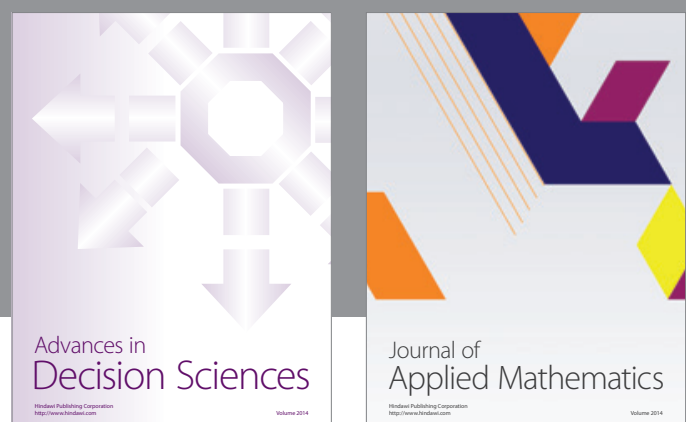

Journal of

Applied Mathematics
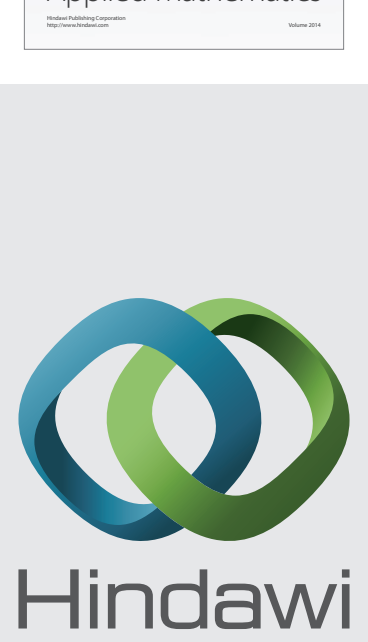

Submit your manuscripts at http://www.hindawi.com
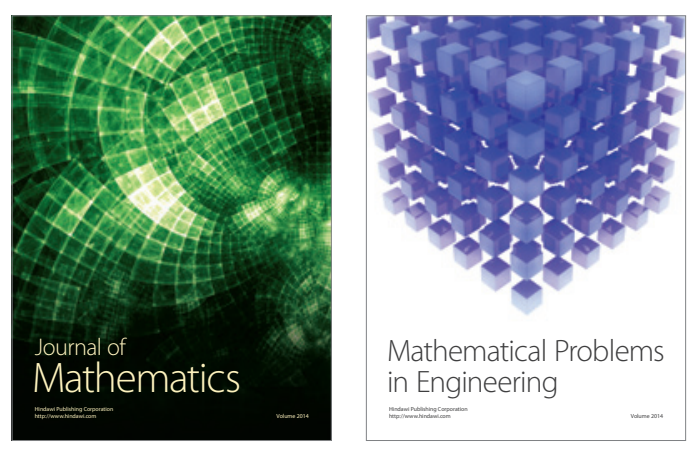

Mathematical Problems in Engineering
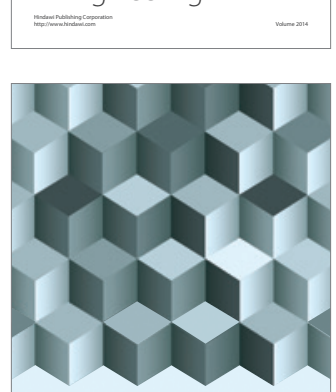

Journal of

Function Spaces
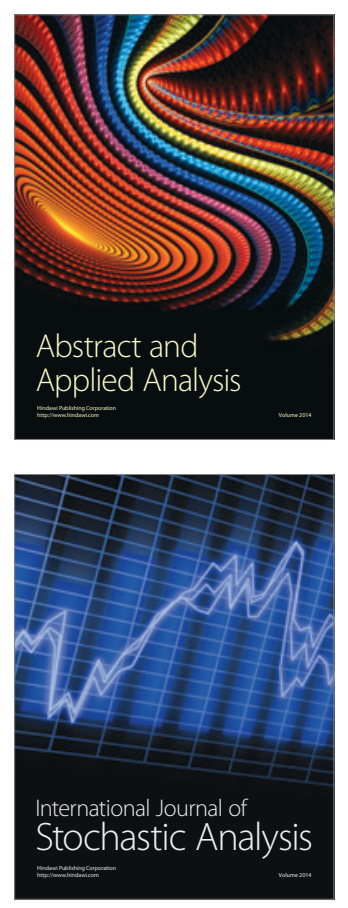

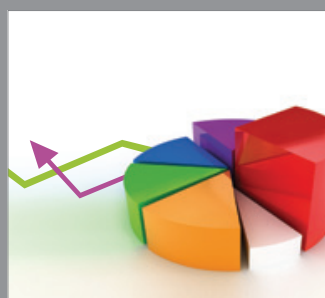

ournal of

Probability and Statistics

Promensencen
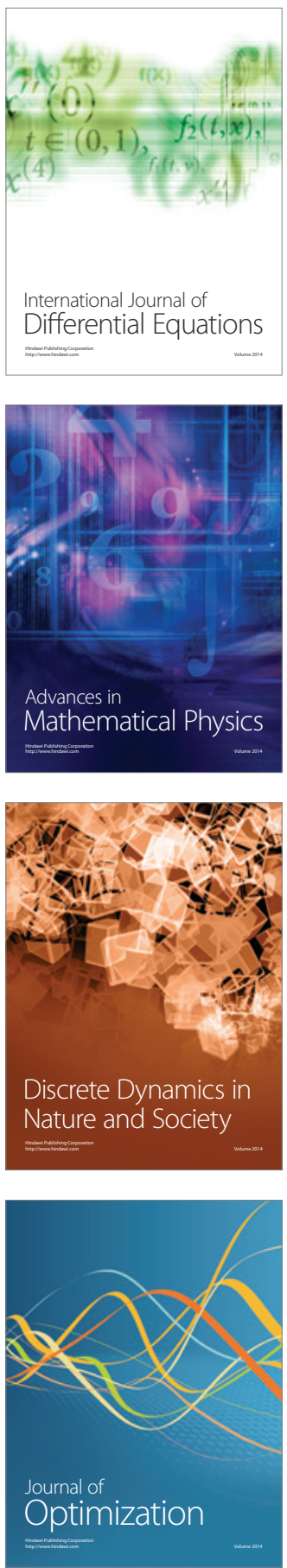